\title{
Comparative genome analysis of multidrug- resistant Pseudomonas aeruginosa JNQH- PA57, a clinically isolated mucoid strain with comprehensive carbapenem resistance mechanisms
}

\author{
Mingju Hao ${ }^{1,2+}$, Wanshan Ma ${ }^{1,2+}$, Xiutao Dong ${ }^{1,2}$, Xiaofeng $\mathrm{Li}^{1,2}$, Fang Cheng ${ }^{1,2}$ and Yujiao Wang ${ }^{1,2^{*}}$
}

\begin{abstract}
Background: The prevalence of clinical multidrug-resistant (MDR) Pseudomonas aeruginosa has been increasing rapidly worldwide over the years and responsible for a wide range of acute and chronic infections with high mortalities. Although hundreds of complete genomes of clinical $P$. aeruginosa isolates have been sequenced, only a few complete genomes of mucoid strains are available, limiting a comprehensive understanding of this important group of opportunistic pathogens. Herein, the complete genome of a clinically isolated mucoid strain $P$. aeruginosa JNQH-PA57 was sequenced and assembled using Illumina and Oxford nanopore sequencing technologies. Genomic features, phylogenetic relationships, and comparative genomics of this pathogen were comprehensively analyzed using various bioinformatics tools. A series of phenotypic and molecular-genetic tests were conducted to investigate the mechanisms of carbapenem resistance in this strain.

(Continued on next page)
\end{abstract}

\footnotetext{
* Correspondence: wangeva-jiao@163.com

†Mingju Hao and Wanshan Ma contributed equally to this work and share the first authorship

${ }^{1}$ Department of Clinical Laboratory Medicine, The First Affiliated Hospital of Shandong First Medical University \& Shandong Provincial Qianfoshan Hospital, Jinan, Shandong, China

${ }^{2}$ Shandong Medicine and Health Key Laboratory of Laboratory Medicine, Jinan, Shandong, China
}

C C The Author(s). 2021 Open Access This article is licensed under a Creative Commons Attribution 4.0 International License, which permits use, sharing, adaptation, distribution and reproduction in any medium or format, as long as you give appropriate credit to the original author(s) and the source, provide a link to the Creative Commons licence, and indicate if changes were made. The images or other third party material in this article are included in the article's Creative Commons licence, unless indicated otherwise in a credit line to the material. If material is not included in the article's Creative Commons licence and your intended use is not permitted by statutory regulation or exceeds the permitted use, you will need to obtain permission directly from the copyright holder. To view a copy of this licence, visit http://creativecommons.org/licenses/by/4.0/ The Creative Commons Public Domain Dedication waiver (http://creativecommons.org/publicdomain/zero/1.0/) applies to the data made available in this article, unless otherwise stated in a credit line to the data. 
(Continued from previous page)

Results: Several genomic features of MDR P. aeruginosa JNQH-PA57 were identified based on the whole-genome sequencing. We found that the accessory genome of JNQH-PA57 including several prophages, genomic islands, as well as a PAPI-1 family integrative and conjugative element (ICE), mainly contributed to the larger genome of this strain (6, 747,067 bp) compared to other popular P. aeruginosa strains (with an average genome size of 6,445,223 bp) listed in Pseudomonas Genome Database. Colony morphology analysis and biofilm crystal staining assay respectively demonstrated an enhanced alginate production and a thicker biofilm formation capability of JNQH-PA57. A deleted mutation at nt 424 presented in mucA gene, resulted in the upregulated expression of a sigma-factor AlgU and a GDP mannose dehydrogenase AlgD, which might explain the mucoid phenotype of this strain. As for the carbapenem resistance mechanisms, our results revealed that the interplay between impaired OprD porin, chromosomal $\beta$ lactamase OXA-488 expression, MexAB-OprM and MexXY-OprM efflux pumps overexpression, synergistically with the alginates-overproducing protective biofilm, conferred the high carbapenem resistance to $P$. aeruginosa JNQH-PA57.

Conclusion: Based on the genome analysis, we could demonstrate that the upregulated expression of alg $U$ and algD, which due to the truncation variant of MucA, might account for the mucoid phenotype of JNQH-PA57. Moreover, the resistance to carbapenem in $P$. aeruginosa JNQH-PA57 is multifactorial. The dataset presented in this study provided an essential genetic basis for the comprehensive cognition of the physiology, pathogenicity, and carbapenem resistance mechanisms of this clinical mucoid strain.

Keywords: Complete genome sequencing, Pseudomosa aeruginosa, Mucoid strain, Alginates-overproducing, Carbapenem resistance mechanisms

\section{Background}

Pseudomonas aeruginosa is an opportunistic pathogen that can cause a wide range of acute and chronic infections. It is also responsible for the life-threatening infections in patients with malignancy, immunosuppression, burns, and those who performed mechanical ventilation [1]. P. aeruginosa possesses versatile abilities to evade host immune response and cause stubborn persistent infections, such as switching to mucoid phenotype, reducing its motility and virulence, presenting a perpetual biofilm state, and developing strong resistance [2, 3]. Even worse, researches showed that the prevalence of clinical multidrug-resistant (MDR) strains has been increasing rapidly worldwide over the years [4-6].

The emergence of carbapenem-resistant $P$. aeruginosa strains greatly compromised the effectiveness of first-line carbapenems treatment. In addition to the acquisition of carbapenem-hydrolyzing $\beta$-lactamases [7], carbapenem resistance in $P$. aeruginsa is known to be the result of interplay between the reduction of permeability, overexpression of the efflux pumps, as well as activity of an inducible chromosomal $\beta$-lactamase AmpC [8]. Furthermore, $P$. aeruginosa is also notorious for the hallmark of conversion to the biofilm-growing mucoid (alginate-producing) phenotype, which makes this pathogen adaptable for long-term persistence in chronic infections [9]. Once the chronic infection is established, $P$. aeruginosa may acquire mutations leading to the conversion of mucoid phenotype, and exhibit reinforced recalcitrance to clearance by the immune system and antimicrobial therapy [9].

The genome size of $P$. aeruginosa commonly varies from $5.5 \mathrm{Mb}$ to $7 \mathrm{Mb}[10,11]$. The incredible metabolic versatility and strong antibiotics resistance of $P$. aeruginosa are generally attributed to genomic diversity [11]. The accessory genomes are usually composed of horizontally transferable elements which include integrative and conjugative elements (ICEs), genomic islands (GIs), prophages, transposons, insertion sequences (ISs), and integrons [12], and they often contribute to the unique physiology, pathogenesis and antibiotics resistances of the corresponding strains $[7,13]$. Although over hundreds of complete genomes of clinical $P$. aeruginosa isolates were deposited in the National Center for Biotechnology Information (NCBI) GenBank, only a few complete genomes of mucoid strains are available (including but not limited to $P$. aeruginosa FRD1 (NZ_CP010555.1) [14], $P$. aeruginosa DK1-NH57388A (NZ_LN870292.1) [15]), limiting a comprehensive understanding of this important group of opportunistic pathogens. A better understanding of the genetic diversity of mucoid $P$. aeruginosa as well as its antibiotic resistance mechanisms is critical to achieving more targeted therapy of chronic lung infections.

The main goal of this study was to determine and analyse the complete genomic sequence of an MDR $P$. aeruginosa strain with mucoid phenotype isolated from respiratory sputum. We sought genomic and phenotypic differences between this mucoid strain and three widely studied reference strains, P. aeruginoas PAO1, PA14 and ATCC 27853. The genomic features of strain JNQH-PA57 were compared with those of three reference strains, and the phenotypic characterizations of colony morphology and biofilm formation of these four strains were performed in vitro. Moreover, the expression levels of $\operatorname{alg} U$ and $\operatorname{alg} D$, which related to alginate biosynthesis in JNQH-PA57 and PAO1, were assessed as 
well. Finally, we conducted a series of phenotypic and molecular-genetic tests to investigate the mechanisms of carbapenem resistance in P. aeruginosa JNQH-PA57. This work represents the detailed genomic comparison of a clinically isolated mucoid $P$. aeruginosa carrying comprehensive multidrug-resistance mechanisms.

\section{Results}

\section{Extended resistance spectrum of mucoid strain $P$. aeruginosa JNQH-PA57}

The $P$. aeruginosa mucoid strain JNQH-PA57 was isolated from a sputum sample of an 86-year-old male patient hospitalized with symptoms of heart failure and pneumonia. This strain was isolated as the causative pathogen of pneumonia which was highly resistant to piperacillin, ceftazidime, piperacillin/tazobactam, imipenem, meropenem, aztreonam. This strain was also resistant to cefepime, ticarcillin/clavulanic acid; but susceptible to amikacin, tobramycin, levofloxacin, ciprofloxacin, and colistin (Table 1).

Detailed test results of the phenotypic screening for carbapenem resistance were shown in Fig. 1. P. aeruginosa JNQH-PA57 showed $\mathrm{a} \geq 5 \mathrm{~mm}$ enlargement in the inhibition zone diameters of the combined discs with PBA or EDTA plus PBA, compared with imipenem or meropenem alone, whereas the combined-disc that used imipenem/meropenem with or without EDTA showed an unapparent difference in the inhibition zone diameters (Fig. 1). These results suggested that JNQH-PA57 was negative for metallo- $\beta$-lactamase production but positive for serine $\beta$-lactamase production.

\section{Genome features and phylogenetic relationship of $P$. aeruginosa JNQH-PA57}

The hybrid genome assembly resulted in a single circular chromosome of $6,747,067$ bp with an average GC content of $66.03 \%$, which is larger than other published complete genomes of popular $P$. aeruginosa strains (with an average genome size of $6,477,604 \mathrm{bp})$ in Pseudomonas Genome Database (PGDB) (Additional file 1: Table S1). A total of 6239 genes were predicted from JNQH-PA57 genome by NCBI Prokaryotic Genome Annotation Pipeline (PGAP) server, which included 6087 coding sequences (CDS) and identified 63 tRNA genes and 12 rRNA operons and 4 ncRNA genes (Table S1).

In order to examine the levels of genetic diversities and phylogenetic relationships between JNQH-PA57 and publicly available $P$. aeruginosa genome, a total of 157 complete genomes of clinical isolates $P$. aeruginosa including PAO1 were downloaded from the NCBI database. These 157 strains were listed in PGDB [16] as a complete genome represent global $P$. aeruginosa clinical collections (PGDB version 20.2, latest update on September 21, 2020). SNP genome alignment showed that a total of $5,020,270$ SNP sites were identified amongst 158 isolates (including JNQH-PA57) (Fig. 2, Additional file 2: Table S2). Phylogenetic tree revealed all strains can be broadly clustered into two major groups with a minor branch containing three P.aeruginosa strains (including a multi-resistant taxonomic outlier PA7) [17] as a third group (Fig. 2). Group 1 is the larger one which includes the two widely studied reference strains PAO1 [18] and ATCC 27853 [19], as well as some notable clinical strains such as LESB58 [20], DK2 [21] and PAK [22]. Group 2 is a smaller one which contains the well-studied highly virulent strain PA14 which has been used extensively to study the contribution of putative virulence factors to disease [23]. It is apparent that $P$. aeruginosa JNQH-PA57 is very closely related to PA14, and both are clustered into the sublineage within group 2 .

Table 1 Minimal inhibitory concentrations (MICs) of some antibiotics for mucoid strain P. aeruginosa JNQH-PA57

\begin{tabular}{llll}
\hline Category & Antibiotics & MIC $\boldsymbol{\mu g} / \mathbf{m l}$ & Resistance \\
\hline Penicillins & Piperacillin & $>256$ & $\mathrm{R}$ \\
Cephalosporins & Ceftazidime & $>128$ & $\mathrm{R}$ \\
& Cefepime & $>32$ & $\mathrm{R}$ \\
Carbapenems & Imipenem & $>16$ & $\mathrm{R}$ \\
& Meropenem & $>32$ & $\mathrm{R}$ \\
Monobactams & Aztreonam & $>128$ & $\mathrm{R}$ \\
Penicillins+ß-Lactamase inhibitor & Piperacillin/Tazobactam & $>256$ & $\mathrm{R}$ \\
& Ticarcillin/Clavulanic Acid & $>128$ & $\mathrm{R}$ \\
Aminoglycosides & Amikacin & $<2$ & $\mathrm{~S}$ \\
Fluoroquinolones & Tobramycin & $<1$ & $\mathrm{~S}$ \\
Polymyxins & Levofloxacin & $<1$ & $\mathrm{~S}$ \\
\hline
\end{tabular}



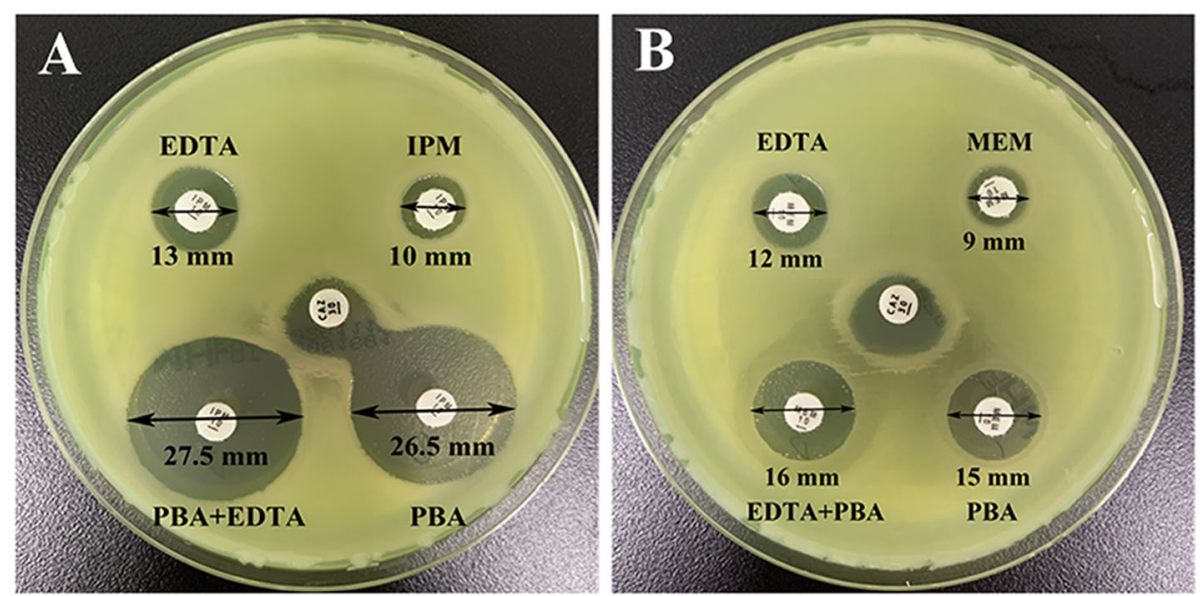

Fig. 1 Identification of the carbapenemase production with three combined-disc tests. a Combined discs consisting of imipenem alone, imipenem with PBA or EDTA respectively, or imipenem with PBA plus EDTA. b Combined discs consisting of meropenem alone, meropenem with PBA or EDTA respectively, or meropenem with PBA plus EDTA. Augmentation of the inhibition zone by $\geq 5 \mathrm{~mm}$ was considered as a positive combined-disc test result

\section{Comparison of $P$. aeruginosa JNQH-PA57 genome with the PAO1, PA14 and ATCC 27853 genomes}

To identify the strain-specific genome features in the JNQH-PA57 genome, the complete genome of JNQHPA57 was further compared with those of three widely studied reference $P$. aeruginosa strains, PAO1 [18], PA14 [24] and ATCC 27853 [19]. Comparative analysis showed that the genome of JNQH-PA57 is larger than that of PA14, PAO1 and ATCC 27853. Several relatively large regions, which consisted of several prophages and GIs, mainly conduce to the large genome of JNQHPA57 (visible in Fig. 3). The genome of JNQH-PA57 shared 676,422 identical sites (86.7\%) with that of PAO1. Even though, the architecture of the JNQH-PA57 genome contains several major differences (Fig. 4). Firstly, the large inversion between ribosomal RNA operons $r r n A$ and $r r n B$ observed in PAO1 [18] does not exist in the genome of $P$. aeruginosa JNQH-PA57 (Fig. 4). Secondly, the latter possesses several large insertion regions $(>50 \mathrm{~kb}$ ) which is absent in PAO1 genome (Fig. 4 and Additional file 3: Table S3). The first insertion is between PA4040 (DUF4340 domain-containing protein) and PA4041 (mandelate racemase), with an integrase in 3 '-end of the sequence, is composed of a $71.85 \mathrm{~kb}$ sequence with a coding capacity for 62 open reading frames (ORFs). Additionally, JNQH-PA57 lacks a 4150 bp fragment containing PA2218, PA2219, and PA2210 genes and has a $52,757 \mathrm{bp}$ insertion downstream of PA2221, which is the second insertion identified in JNQH-PA57 genome. The third insertion between PA1965 and PA1964 (located between the 3,577,938 bp and 3,636,345 bp in JNQH-PA57 genome), which is composed of a $58.4 \mathrm{~kb}$ fragment with a coding capacity for 77 ORFs, is identified as a prophage specifically apparent in the genome of JNQH-PA57, which does not exist in the genomes of PAO1, PA14 and ATCC 27853. Further aligning this insertion with the NCBI database revealed that this fragment existed in the JNQH-PA57 uniquely. The fourth, as well as the largest insertion region, is a 117,766 bp fragment located between PA4541 (tpsA4) and PA4542 (clpB), flanked by 46 bp identical attL and attR sequences. This insertion fragment displays a significant similarity to PAPI-1 family ICEs (Fig. 4).

\section{Gls and prophages identified in the genome of JNQH-PA57}

We predicted the GIs in the JNQH-PA57 genome with the Island Viewer 4 server [25]. A total of nine GIs were identified in the genome of JNQH-PA57 by the Island Viewer 4 server (Table 2). The lengths of these GIs range from 11,253 bp to 37,691 bp. Most of the genes located in the genomic islands encode integrases, transposases (including IS110, IS3 and IS4 family transposases), virulence factors (such as the two-partner secretion system exoprotein TpsA1, type-IV pilus biogenesis and extracellular protein secretion related proteins PilB, PilD, and type III secretion system-related proteins FliR, FliQ, FliP) (Table 2 and Additional file 4: Table S4), which may be important for the survival, nosocomial spread and pathogenicity of the JNQH-PA57.

In addition, four prophages in the genome of $P$. aeruginosa JNQH-PA57, designated as Prophage 1-4, have been predicted using PHASTER [26] (Fig. 3 and Table 3). Prophage 1 is a 30,651 bp fragment located between H5409_03200 and H5409_03390. This prophage is highly conserved in all available $P$. aeruginosa genomes, based on the PHASTER database [26]. Prophage 2 is the largest prophage in this strain with a size of $58,408 \mathrm{bp}$, in which most ORFs encode phage-related proteins such as phage head and tail, transposases and integrases (Additional file 5: Table S5). Prophage 3 is a $13,578 \mathrm{bp}$ 


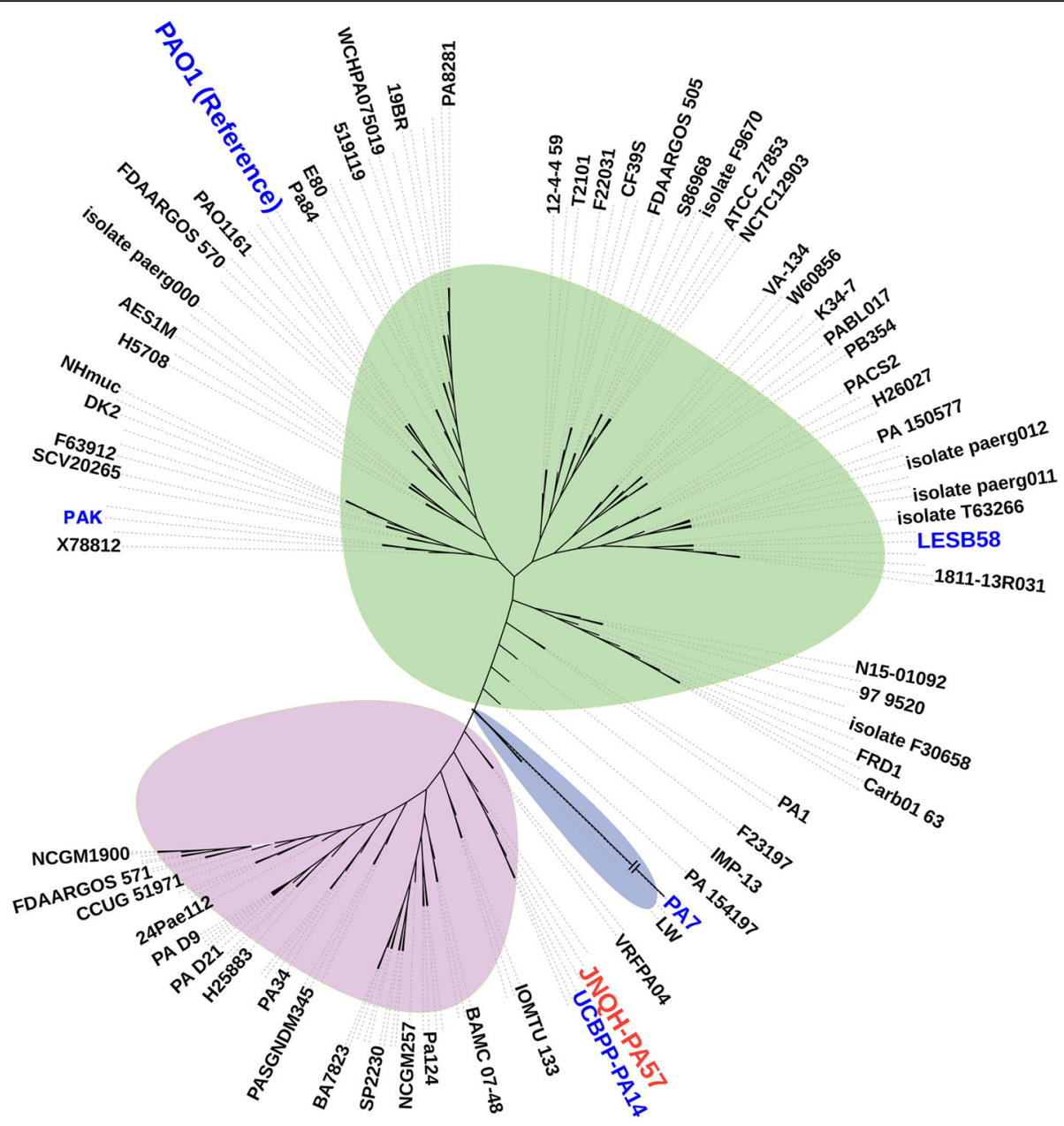

Tree scale: 1

Fig. 2 Unrooted phylogenomic tree of clinically isolated 158 P. aeruginosa (including the JNQH-PA57). The phylogenomic tree was constructed based on identifying SNPs between the reference PAO1 genome and other 157 clinically isolated $P$. aeruginosa genomes. Strains are clustered into three groups (group 1: green, group 2: pink and group 3: blue). Original appearance of this tree was present at the bottom in which the scale bars represent the number of substitutions per site

fragment located between H5409_23135 and H5409_ 23230, which shares high similarity with Pseudomonas phage Pf1, a filamentous bacteriophage identified by Hill et al. [27]. The last prophage is regarded as an incomplete prophage with a very low predicted score, which contains only ten phage hit proteins.

\section{JNQH-PA57 genome contains a PAPI-1 family ICE}

Based on the sequence alignment and ICEberg database [28], a 117,766 bp putative ICE, with the type IV secretion system (T4SS), is located on the chromosome (between the position $5,424,238 \mathrm{bp}$ to $5,542,003 \mathrm{bp}$ ) of this strain (Fig. 4) and bracketed by two 46 bp highly conserved direct repeats (5'-ATGGTGGGTCGTGTAGGA
TTCGAACCTACGACCAATTGGTTAAAAG-3'), named attL and attR, on both sides. This element, named ICE $_{\text {JNPA57, }}$, which is located adjacent to a tRNA cluster integration site, starts with a gene encoding ParA family protein and ends with a site-specific recombinase (Fig. 5). The sequence and architecture of operons flanking the putative integration site in this putative ICE showed significant similarities with those in the PAPI-1 family ICEs [13], especially with pathogenicity islands pKLC102 in P. aeruginosa clone C strains (with 61\% coverage and $96.14 \%$ identity) [29] and the pathogenicity island PAPI-1 in P. aeruginosa PA14 (with 63\% coverage and $97.95 \%$ identity) [30] (Fig. 5). Comparison between these three PAPI-1 family ICEs revealed that the 




conjugation machinery elements which contain the T4SS, as well as genes those encoding putative ICE family proteins, relaxases and site-directed integrase, were highly conserved in these three ICEs, especially between the $\mathrm{ICE}_{\text {INPA57 }}$ in JNQH-PA57 and PAPI-1 in PA14 (Fig. 5). In addition, 121 ORFs were predicted in ICE JNPA57, of which at least 30\% were "hypothetical proteins" (Additional file 6: Table S6). It was noted that two segments, which contained several cargo genes encoding the putative metallohydrolases and multidrug transporters respectively, were exclusively existed in the $\mathrm{ICE}_{\mathrm{JNPA57}}$ (Fig. 5).
Clusters of orthologous groups comparison

Comparison of Clusters of Orthologous Groups (COG) annotations revealed a total of 25 COGs are exclusively present in $P$. aeruginosa JNQH-PA57 compared with PAO1, PA14 and ATCC 27853 (Fig. 6 and Additional file 7: Table S7). Most of these COGs are efflux transporter related proteins, which might contribute to the extended resistance spectrum exhibited by this strain. The details of the COG communities' distribution among these four strains were exhibited in Fig. 6. Of note, JNQH-PA57 shares a total of 2365 COGs with PA14 (Fig. 6), a much 


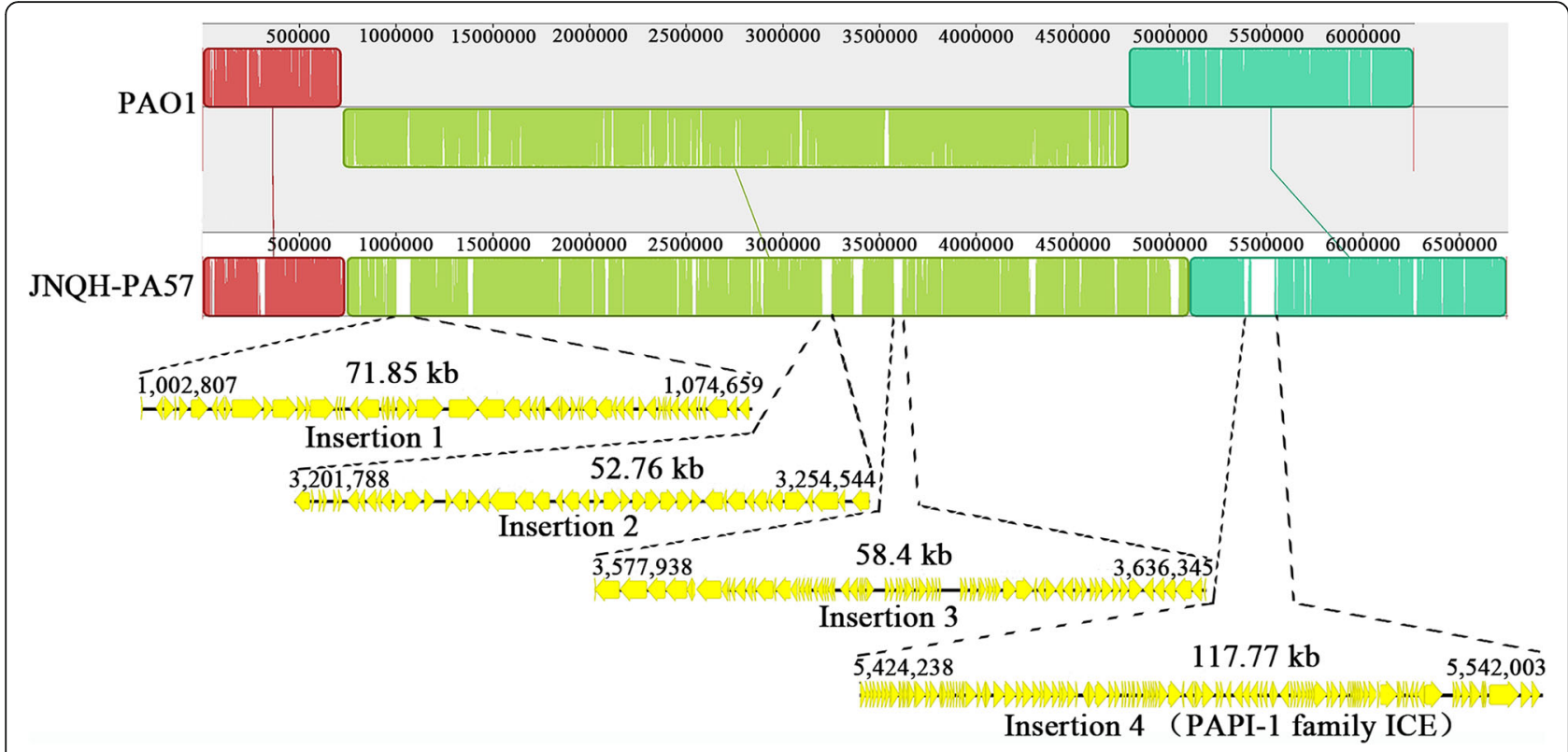

Fig. 4 Mauve alignment of JNQH-PA57 and PAO1 revealed major structural variations between the genomes of these two strains. Blocks indicated regions with percentage of nucleotide sequence identity higher than 75\%. The inversion between $r$ rnA and $r$ rnB rRNA operons was colored in aqua. Bottom panel indicated positions and schematic gene organization of four large insertion regions ( $>50 \mathrm{~kb}$ ) which were absent in PAO1 genome

higher number than that with PAO1 (shares 2340 COGs) and ATCC 27853 (shares 2308 COGs). These shared COGs also echoed the phylogenetic relationship as described above, that JNQH-PA57 has a rather closer genetic relationship with PA14 than with PAO1 and ATCC 27853.

\section{P. aeruginosa JNQH-PA57 exhibits distinctive biofilm-} growing mucoid (alginate-producing) colony morphology due to the derepression of AlgU

The colony morphology experiment showed that a distinctive mucoid colony was observed in JNQH-PA57 but not in PAO1, PA14 and ATCC 27853, revealing an alginate overproduction from the former strain (Fig. 7a).
The expression analysis showed that the transcriptional levels of both $\operatorname{alg} U$ and $\operatorname{algD}$ in the JNQH-PA57 were significantly higher than those in the nonmucoid reference strain PAO1 (Fig. 7b). The up-regulation in the transcription of the alginate biosynthetic operon might explain mucoid phenotype of this strain. Specifically, the SNP distribution analysis revealed that the presence of a deleted mutation at nt 424 in $P$. aeruginosa JNQH-PA57 $m u c A$ gene, resulting in a frameshift variant, with the formation of a premature stop codon, likely account for the overproduction of alginate and further leading to the mucoid phenotype of this strain. Biofilm formation and growth curve analysis showed that mucoid strain JNQH-

Table 2 List of Gls identified in P. aeruginosa JNQH-PA57

\begin{tabular}{|c|c|c|c|c|c|}
\hline ID & Start & End & Size (bp) & Gene locus tag rang & Genes of interest \\
\hline Gl 1 & 713,078 & 724,317 & 11,239 & H5409_03350- H5409_03400 & $\begin{array}{l}\text { Prophage 1, anthranilate phosphoribosyltransferase TrpC, } \\
\text { indole-3-glycerol phosphate synthase TrpD }\end{array}$ \\
\hline Gl 2 & 998,362 & $1,034,278$ & 35,916 & H5409_04670- H5409_04810 & Hypothetical proteins, IS110 family transposase \\
\hline Gl 3 & $1,376,175$ & $1,397,681$ & 21,506 & H5409_06435- H5409_06535 & VapC family toxin, conjugative transfer protein TrbJ, TrbL \\
\hline Gl 4 & $2,864,029$ & $2,901,720$ & 37,691 & H5409_13445- H5409_13535 & $\begin{array}{l}\text { Proteins involved in iron acquisition and virulence, IS4 } \\
\text { family transposase, IS3 family transposase }\end{array}$ \\
\hline Gl 5 & $4,885,149$ & $4,896,402$ & 11,253 & H5409_22590-H5409_22650 & tyrosine-type recombinase/integrase, adenylyltransferase \\
\hline Gl 6 & $5,386,356$ & $5,411,738$ & 25,382 & H5409_24840- H5409_24980 & $\begin{array}{l}\text { site-specific integrase, pilin, pilus assembly ATPase PilB, } \\
\text { prepilin peptidase PilD }\end{array}$ \\
\hline Gl 7 & $5,428,676$ & $5,440,617$ & 11,941 & H5409_25065- H5409_25160 & Integrative conjugative elements \\
\hline Gl 8 & $5,692,873$ & $5,707,176$ & 14,303 & H5409_26330- H5409_26415 & $\begin{array}{l}50 S \text { ribosomal protein, IS3 family transposase, type II } \\
\text { toxin-antitoxin system ReIE/ParE family toxin }\end{array}$ \\
\hline Gl 9 & $6,261,122$ & $6,277,771$ & 16,649 & H5409_28890-H5409_29015 & Type III secretion system related proteins FliR, FliQ, FliP \\
\hline
\end{tabular}


Table 3 List of prophages identified in P. aeruginosa JNQH-PA57

\begin{tabular}{llllllll}
\hline Prophage ID & Region Length & Completeness & Score & Total Proteins & Region Position & Close Phage & GC \% \\
\hline Prophage 1 & $30.6 \mathrm{~kb}$ & intact & 150 & 37 & $691,434-722,084$ & Pseudomonas phage phiCTX & $64.15 \%$ \\
Prophage 2 & $57 \mathrm{~kb}$ & intact & 120 & 58 & $3,579,267-3,636,345$ & Pseudomonas phage vB_PaeP_Tr60_Ab3 & $61.29 \%$ \\
Prophage 3 & $13.5 \mathrm{~kb}$ & intact & 96 & 17 & $4,996,552-5,010,118$ & Pseudomonas phage Pf1 & $56.24 \%$ \\
Prophage 4 & $9.4 \mathrm{~kb}$ & incomplete & 10 & 16 & $5,424,375-5,433,867$ & Bacill_phBC6A51 & $61.57 \%$ \\
\hline
\end{tabular}

PA57 formed a more robust biofilm whereas exhibited an attenuate planktonic growth compared to PAO1, PA14 and ATCC 27853 (Fig. 7c and d).

Multiple factors contribute to carbapenem resistance in $P$. aeruginosa JNQH-PA57

Antimicrobial resistance (AMR) genes searching results showed that JNQH-PA57 harbors 47 genes related to antibiotic and disinfectant resistance, most of which (39 genes) are efflux pump-related genes. Six are antibiotic inactivation genes and only two of them are antibiotic target alternation genes (Fig. 3 and Additional file 8: Table S8). Remarkably, JNQH-PA57 possesses 2 chromosomal $\beta$-lactamase genes, $b l a_{\mathrm{PDC}-12}$ and $b l a_{\mathrm{OXA}-488}$.

To study whether the carbapenem resistance phenotype of this strain was associated with $\mathrm{OprD}$ mutations, nucleotide and amino acid sequences of this porin in JNQH-PA57 were blasted with the OprD in PAO1. Ten non-synonymous mutations were presented in OprD of JNQH-PA57, including T103S, K115T, F170L, E185Q, P186G, V189T, R310E, A315G, P417* (" represents a stop codon) and G425A.

The expressions of two major resistance-nodulationdivision (RND) family efflux pumps (MexAB-OprM and
MexXY-OprM) were investigated in this study used $\operatorname{mexB}$ and mex $Y$ as target genes, respectively. The transcription levels of mexB and mexY in JNQH-PA57 significantly increased 18.75-and 9.75-fold, compared to those in the reference strain PAO1 (which is sensitive to carbapenem) (Fig. 8).

\section{Discussion}

Having a repertoire of highly flexible genes, the opportunistic pathogen $P$. aeruginosa shows a great deal of diversity thriving in different habitats [31]. The extensive genome plasticity of this pathogen, in large part, is provided by pathogenicity islands [31]. One of the wellcharacterized pathogenicity islands PAPI-1 is an ICE that encompasses a number of virulence determinants [30]. In addition to the PAPI-1 first described in P. aeruginosa PA14, several different ICEs that belonged to PAPI-1 family have been identified in abundant Pseudomonas genomes and confer multiple adaptive functions such as antibiotics and heavy metal resistance to the hosts $[13,32]$. In the current study, a novel ICE JNPA57 was identified in the genome of JNQH-PA57 isolated from a clinical source. ICEs are mobile genetic elements, which harbor specific modular structures encoding the

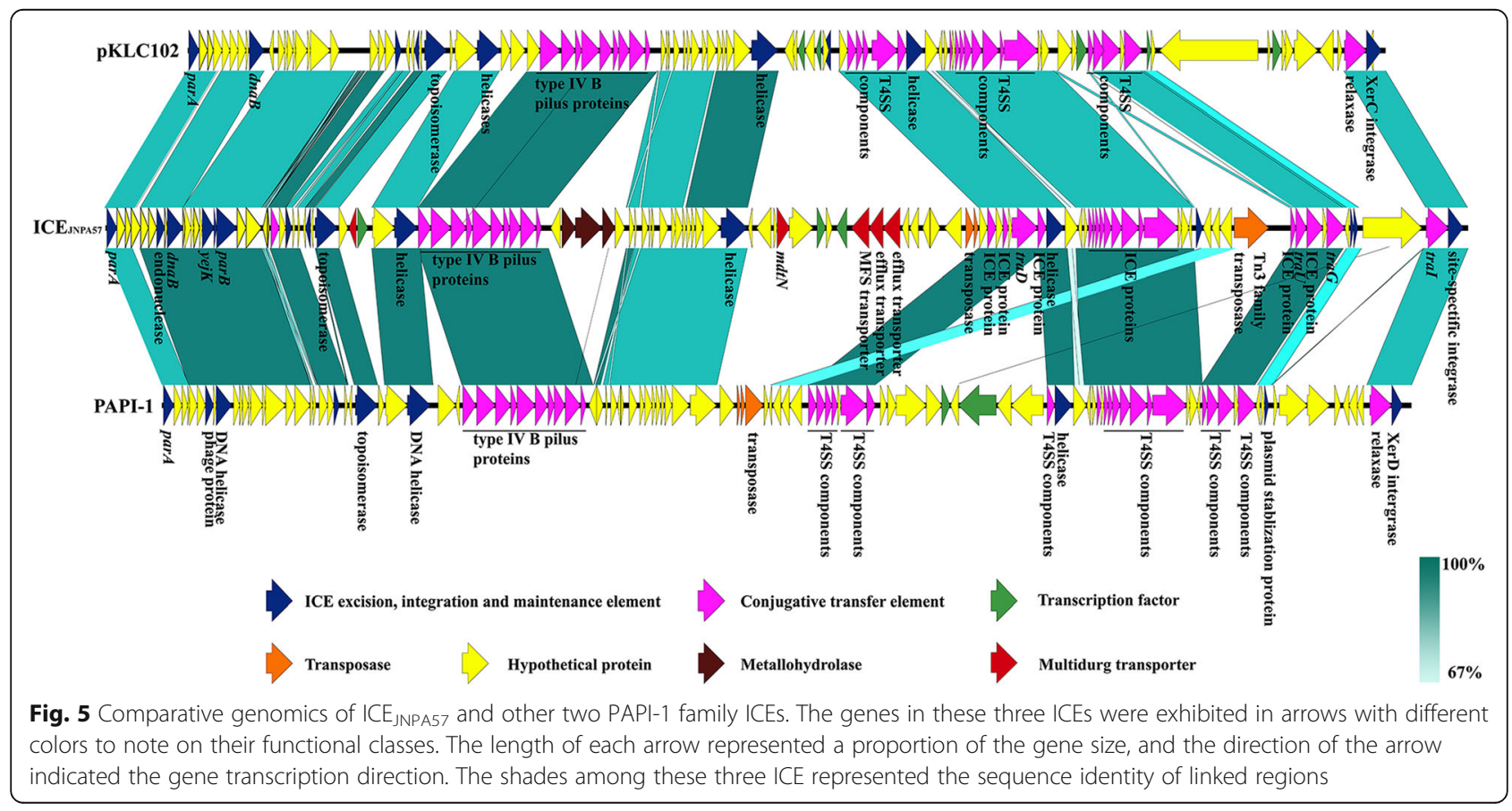




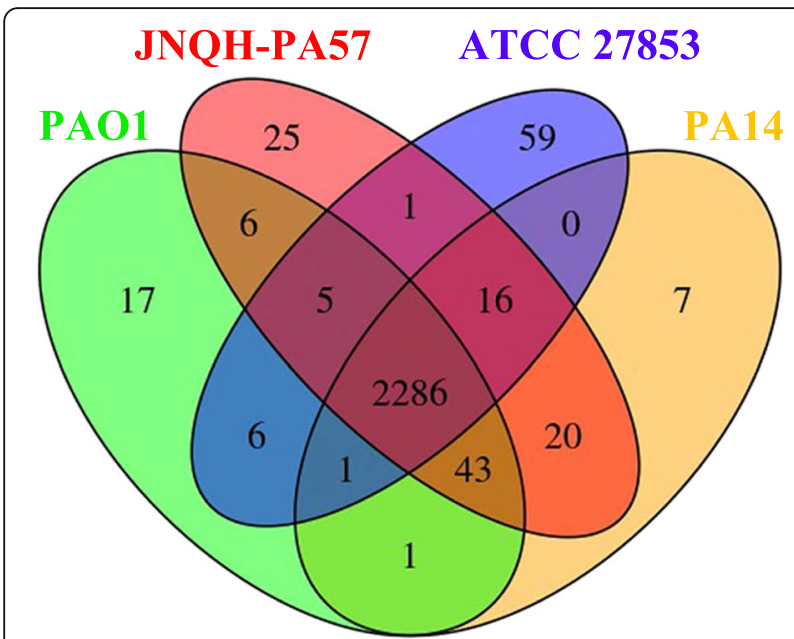

Fig. 6 COG comparisons of $P$. aeruginosa strains. Venn diagram showed the number of exclusive and shared genes among four $P$. aeruginosa strains: PAO1, JNQH-PA57, ATCC 27853, and PA14. The number within the ellipses in the venn diagram represent the number of unique genes, those shared among two, three and all four strains of PAO1, JNQH-PA57, ATCC 27853, PA14 strains were based on the COG gene annotations

complete conjugation machinery (mainly the T4SS) [33]. A previous study revealed that a cluster of ten genes encoded in PAPI-1 was responsible for the synthesis and assembly of the T4SS [34]. This cluster of 10 genes encoding the T4SS was also found to be embedded in the ICE $_{\text {JNPA57. Moreover, this T4SS encoding operon in }}$ ICE $_{\text {INPA57 }}$ shows highly conservation with (up to $98.5 \%$ identity) with that in PAPI-1. The integration and excision of ICE into/out of the host chromosome, which relies on an ICE encoded site-directed integrase, is another defining feature of ICEs [33]. Here, we found that the attL and attR direct repeats flanked by this $\mathrm{ICE}_{\mathrm{INPA} 57}$ resemble the conserved integration site in almost all PAPI-1 family ICEs [13]. Besides, the boundary operons organization of $\mathrm{ICE}_{\text {JNPA57, }}$, which starts with a ParA family protein and ends with a putative relaxase (TraI) as well as a sitespecific recombinase (Int), are also highly conserved with other PAPI-1 family ICEs [13]. These proteins located at both sides of the ICE are respectively correlated with the mobilization and integration of pathogenicity GIs [35, 36]. All these pieces of evidence mentioned above indicated a close evolutionary relationship between PAPI-1 in PA14 and $\mathrm{pKLC102}$ in clone $\mathrm{C}$ strains, revealing that the $\mathrm{ICE}_{\text {INPA57 }}$ identified in our study should be a member of the PAPI-1 family ICEs.

$P$. aeruginosa JNQH-PA57 studied here is a clinical strain with mucoid phenotype. The conversion of $P$. aeruginosa to mucoid alginate-overproducing form is a critical persistence factor of the inextirpable chronic infection caused by this pathogen [37]. The mucoid switch usually results from bereft regulation of the sigma factor
$\mathrm{AlgU}$ via inactivating in the anti-sigma factor MucA [37]. MucA is a negative regulator for AlgU (a sigma factor that was required for expression of the key alginate biosynthetic gene $\operatorname{alg} D$ ) expression, and responsible for preventing AlgU from binding and activating target promoters. Once MucA is inactivated via nonsense and frameshift mutations, the liberated AlgU will activate the genes related to alginate biosynthesis, such as $\operatorname{alg} D$ (encoding a GDP mannose dehydrogenase in the alginate biosynthesis operon), resulting in constitutive production of alginate [37]. The mutation can occur throughout the mucA gene, which, in turn, may result in the generation of MucA proteins of different sizes [38]. Herein, we found that MucA variant in JNQH-PA57 is resulted from an nt 424 deletion mutation, leading to the generation of a protein only containing the N-terminal 147 amino acids of MucA (losing almost 50 amino acids compared with the wild type MucA protein). Yin et al. have reported that mucoid phenotype in clinical isolates can be not only modulated by the size of the MucA protein but also influenced by the genotype of the alg $U$ in a particular strain [38]. The size of MucA determines the cellular localization of this protein and its ability to repress $\mathrm{AlgU}$, while the genotype of the algU determines the activity of AlgU [38]. However, no non-synonymous mutations were detected in JNQH-PA57 algU gene. Consequently, these results indicated that the mucoidy in this clinical isolate JNQH-PA57 was likely to be driven by the truncation variant of MucA protein.

Additionally, the alginate overproduction mucoid strain would form a thicker biofilm with large extended mushroom-like microcolonies compared to wild-type strains [39]. It was regarded that this mucoid microcolony mode of growth provides a major benefit to chronic colonization of $P$. aeruginosa in the cystic fibrosis (CF) lung, due to the surrounding overproducing alginate, which would protect the bacteria from the host immune system clearance by restraining phagocytosis, withstanding oxidative burst, interfering opsonization and providing an immunomodulatory role [40, 41]. Moreover, the mushroom-like biofilm had been reported to display an up to 100 to 1000 -fold higher tolerance to antibiotics compared with the planktonic bacteria [42]. On the other hand, biofilm growth in cystic fibrosis lungs is associated with the slow growth of $P$. aeruginosa [43]. In our case, we observed that the mucoid JNQH-PA57 formed a more robust biofilm, whereas exhibited an attenuate growth compared with other nonmucoid strains. The increasing doubling time and low bacterial metabolic activity of $P$. aeruginosa in the biofilm-growth mode are partly responsible for the tolerance to some antibiotics [43].

Pseudomonas-derived cephalosporinase (PDC) is a class C beta-lactamase, which confers Pseudomonas strains 


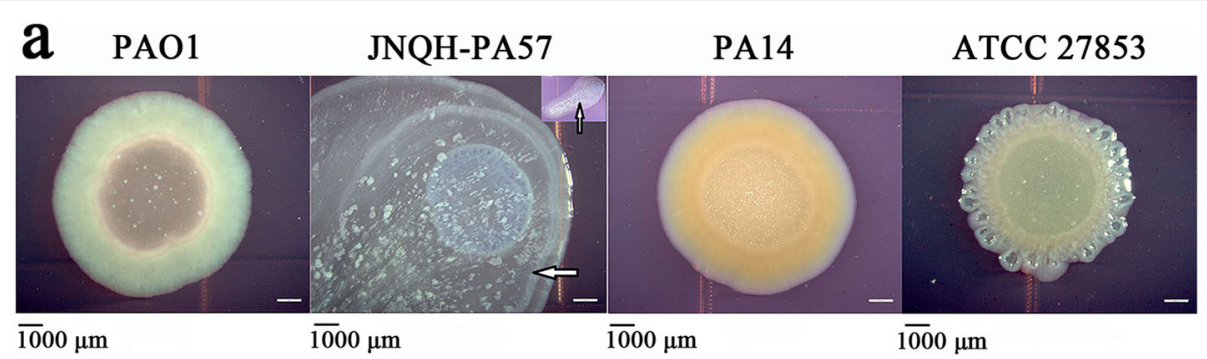

b

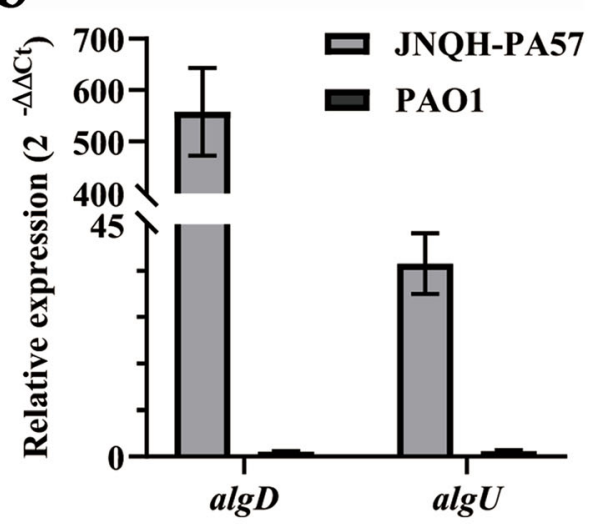

C

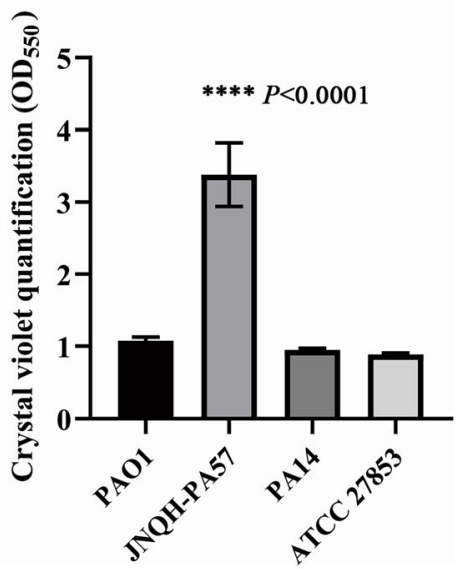

d

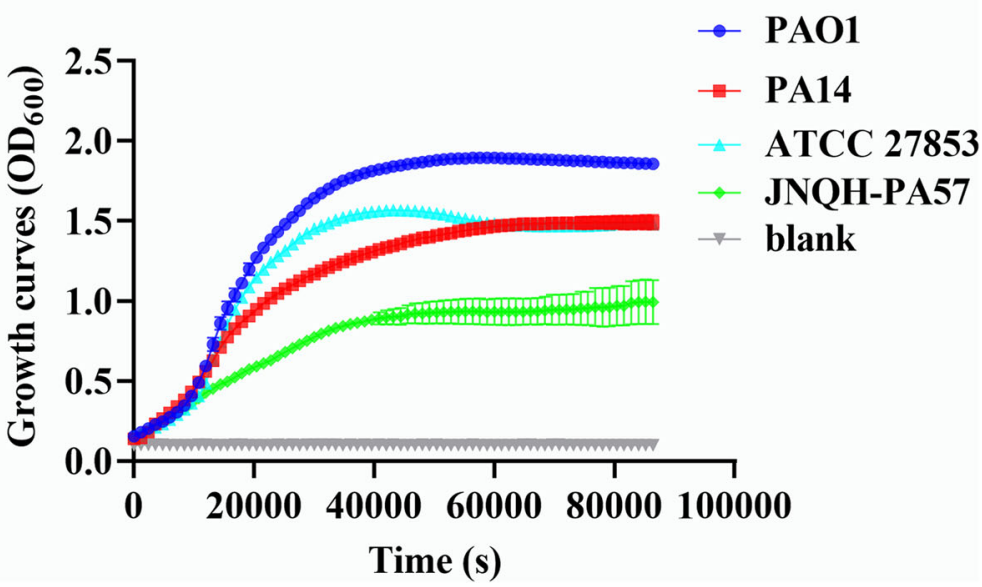

Fig. 7 Colony morphology, alginate biosynthesis related genes expression and biofilm formation of $P$. aeruginosa JNQH-PA57. a Colony morphology of four $P$. aeruginosa strains cultured at $25^{\circ} \mathrm{C}$ on LB agar plates supplemented with Coomassie blue and Congo Red. The white arrow points to the exopolysaccharide produced by JNQH-PA57. b Expression of algD and algU genes in JNQH-PA57 and PAO1 measured by qRT-PCR. Each experiment was done in triplicate. $\mathbf{c}$ Biofilm formation of four $P$. aeruginosa strains via crystal violet staining. $\mathbf{d}$ Comparison of the growth of $P$. aeruginosa JNQH-PA57 and three other $P$. aeruginosa reference strains on LB. Representative growth curves of JNQH-PA57, PAO1, PA14 and ATCC 27853 were measured via microplate method

resistance to all beta-lactams except carbapenems [44]. The $\beta$-lactamase OXA-488 is an OXA-50 family $\beta$ lactamase, which can only hydrolyze imipenem at a low level and do not significantly hydrolyze meropenem [45]. Although these two $\beta$-lactamase encoding genes, including bla $_{\mathrm{PDC}}-12$ and $b l a_{\mathrm{OXA}-488}$, were detected in JNQH-
PA57, they are unlikely to be the dominant factor that significantly influences the carbapenem resistance phenotype of this strain. Studies have demonstrated that permeability reduction of the outer membrane protein OprD was one of the predominant mechanisms of imipenem resistance in $P$. aeruginosa [46, 47]. OprD-mediated carbapenem 


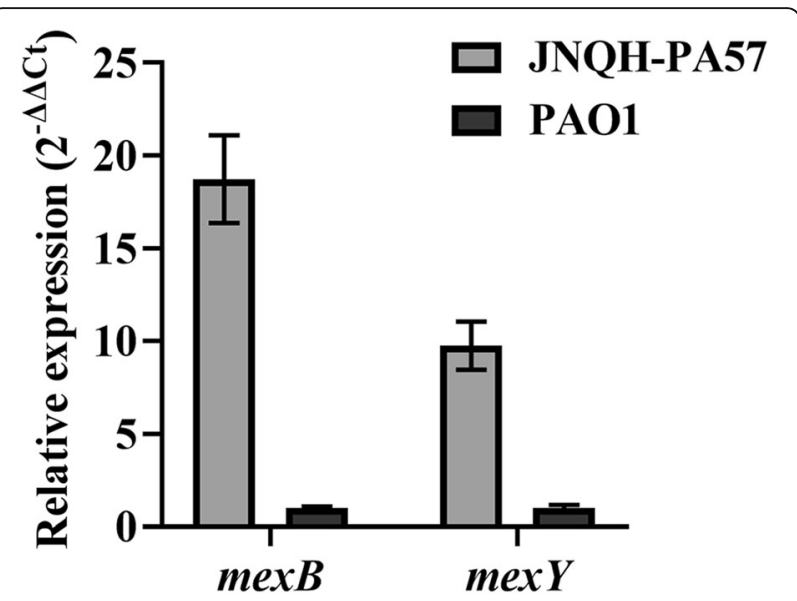

Fig. 8 Expression of mexB and mexY genes in JNQH-PA57 and PAO1 measured by qRT-PCR. Each experiment was done in triplicate

resistance can result from the reduced expression of oprD or the inactivation of this porin through insertion/deletion mutation and/or a premature stop codon [46, 48]. P. aeruginosa clinical strains, which carrying an OprD with polymorphisms, particularly the F170L substitution, were found to rapidly develop carbapenem resistance [48]. The F170L mutation is located on loop3 of oprD-encoding porin, which, along with loop2, is related to imipenem binding and passage through the porin [49]. Notably, this F170L polymorphism alone was able to contribute to the carbapenem-resistance phenotype in $P$. aeruginosa [48]. In our study, ten non-synonymous mutations (including but not limited to F170L and a premature stop codon presented at $\mathrm{N}$-terminal 417 amino acid site) were observed in OprD of JNQH-PA57. We inferred that the amino acid substitution of F170L and truncation of OprD due to a G > A base substitution at nt 1251 in oprD gene might confer imipenem resistance in JNQH-PA57.

Except for AmpC hyperproduction and OprD inactivation, clinical resistance to carbapenems is regarded to require additional mechanisms, such as RND family efflux pumps (especially MexAB-OprM) overexpression [50, 51]. The RND efflux pump MexAB-OprM, which possesses the broadest substrate profile for multiple classes of antibiotics, promotes the extrusion of various antibiotics [50]. Overexpression of this efflux pump had been frequently detected in a good deal of clinical MDR P. aeruginosa isolates [52, 53]. Previous studies indicated that MexAB-OprM is capable of exporting $\beta$-lactams including meropenem [54]. However, this efflux pump cannot export imipenem because of the lack of a specific heterocyclic side chain in imipenem that is recognized by MexAB-OprM $[53,54]$. On the other hand, it has been reported that overexpression of MexAB-OprM was always combined with the increased expression of MexXY-OprM in the carbapenem-resistant $P$. aeruginosa strains [8]. The isolates with the latter efflux pump profile were resistant or with intermediate resistance to both imipenem and meropenem [8]. We obtained the same results with these previous reports: both MexAB-OprM and MexXY-OprM efflux pumps were overexpressed in this carbapenem-resistant strain JNQH-PA57.

\section{Conclusion}

In summary, several genomic features of $P$. aeruginosa JNQH-PA57 were identified based on the whole-genome sequencing. Phylogenetic analysis showed that JNQH-PA57 has a high genetic relationship with PA14. Comparative genomic analysis revealed that JNQH-PA57 possesses several genomic islands, prophages, as well as a PAPI-1 family ICE, which are absent in the genome of PAO1. JNQHPA57 presented a distinctive alginate overproduction mucoid phenotype. A deleted mutation at nt 424 resulting in a frameshift variant of muсA gene in JNQH-PA57, further led to the upregulated expression of $\operatorname{alg} U$ and $\operatorname{alg} D$, might account for the mucoid phenotype of this strain. Finally, multiple factors were found to contribute to carbapenem resistance in $P$. aeruginosa JNQH-PA57. It was speculated that the interplay between impaired OprD porin, overexpression of the MexAB-OprM and MexXY-OprM efflux pumps, and chromosomal $\beta$-lactamase OXA-488 expression, synergistically with the alginates-overproducing protective biofilm, conferred the high carbapenem resistance to $P$. aeruginosa JNQH-PA57. The dataset presented in this study provided an essential genetic basis for the comprehensive cognition of the physiology, pathogenicity, and antibiotics resistance mechanisms of this multidrug-resistant $P$. aeruginosa mucoid strain.

\section{Methods}

Ethics

The current study focused only on characterizing an MRD clinical isolate $P$. aeruginosa with mucoid phenotype. This isolate was retrospectively retrieved from the Bacteria Bank hosted in the Department of Laboratory Medicine, the First Affiliated Hospital of Shandong First Medical University Hospital, Jinan. Detail clinical information of the patient was not required in this study. Since the microbial culture had been ordered by physicians because of their necessity for clinical management, the patient's informed consent was not required and not collected. The Ethics Committee of the First Affiliated Hospital of Shandong First Medical University exempted this study from review and the Review Board also waived the requirement for informed consent.

\section{Bacterial strain, culture conditions, and antibiotic susceptibility tests}

The isolated JNQH-PA57 was grown in Mueller-Hinton agar (MHA) (Oxoid, Hampshire, United Kingdom) at $37^{\circ} \mathrm{C}$ for $24 \mathrm{~h}$. The species identification was determined with Microflex LT/SH MALDI-TOF mass spectrometer 
(Bruker, Germany). The antimicrobial susceptibility of this stain was performed using MIC evaluation via the E-test method for the following antimicrobial agents: piperacillin, piperacillin/tazobactam, ticarcillin/clavulanic acid, ceftazidime, cefepime, aztreonam, imipenem, meropenem, amikacin, tobramycin, levofloxacin, ciprofloxacin, according to the manufacturer's guidelines $(\mathrm{AB}$ Biodisk, Sweden). For colistin, the MIC was determined via a broth microdilution method, according to the Clinical and Laboratory Standards Institute (CLSI) guideline. P. aeruginosa ATCC 27853 served as a quality control strain for susceptibility testing. The interpretation of the results was based on the CLSI 2020 guideline [55].

\section{Phenotypic tests for carbapenemase detection}

A phenotypic carbapenemase assay compared carbapenem activity with and without the presence of inhibitors as previously reported [56], with slight modification. Briefly, combined-disc tests of imipenem/meropenem alone and with $400 \mu \mathrm{g}$ of PBA or $292 \mu \mathrm{g}$ of EDTA or both of PBA and EDTA were assessed for the identification of the different type of carbapenemase production. PBA was dissolved in DMSO at a concentration of $20 \mathrm{mg} / \mathrm{mL}$. Anhydrous EDTA was dissolved in distilled water at a concentration of $0.1 \mathrm{M}$. $20 \mu \mathrm{L}$ PBA solution (containing $400 \mu \mathrm{g}$ of PBA) and $10 \mu \mathrm{L}$ EDTA solution (containing $292 \mu \mathrm{g}$ of EDTA) were dispensed onto commercially available imipenem/meropenem discs respectively or simultaneously. The phenotypic assay was performed by inoculating the bacterial suspension onto the MHA. Then the MHA plates were incubated at $37^{\circ} \mathrm{C}$ overnight. The diameter of the growth inhibitory zone around four imipenem/meropenem discs was measured and compared. Enlargement of the inhibition zone by $\geq 5$ $\mathrm{mm}$ was considered as a positive combined-disc test result.

\section{Bacterial DNA extraction, genome sequencing and annotation}

A single colony of $P$. aeruginosa JNQH-PA57 grown on Columbia blood plate (Hapo, China) was inoculated into 5 $\mathrm{mL}$ of LB medium and shaken at $180 \mathrm{rpm}$ at $37^{\circ} \mathrm{C}$ for $18 \mathrm{~h}$. Bacterial cells were collected by centrifugation and the genomic DNA was extracted with the genomic DNA purification kit (Wizard, USA) according to the manufacturer's instruction, and the DNA integrity was checked on the agarose gel. Next, two independent genomic DNA libraries were prepared for Illumina and Oxford nanopore systems. The combination of long-read Nanopore minION and short-read Illumina NovaSeq 6000 platforms were used to generate the complete genome sequence of $P$. aeruginosa JNQH-PA57. The hybrid genome assembly was performed with unicycler v0.4.8, which allows for both short Illumina reads (accurate) and long Nanopore reads (less accurate) to be used in the conservative mode [57]. The short Illumina reads with high accuracy $(\mathrm{Q} 30>85 \%)$ were aligned against the long Nanopore reads (as a reference), to correct random sequencing errors and then generate a genome assembly of high accuracy. Then the accurate short Illumina reads were aligned with software Bowtie 2 and the final assembly was polished with the Pilon for several rounds to reduce the rate of mismatches and small insertions/deletions [58]. The final genome consensus sequence resulted in one circular replicon of 6,747,067 bp. The complete genome sequence of $P$. aeruginosa JNQH-PA57 was annotated with NCBI Prokaryotic Genome Annotation Pipeline (PGAP), using the method of best-placed reference protein set (GeneMarkS-2+ v4.12) [59]. tRNA genes and rRNA genes were predicted utilizing tRNAscan-SE [60] and RNAmmer [61], respectively. The nucleotide sequence of JNQH-PA57 has been submitted and deposited in the NCBI Nucleotide database (with the accession number of NC_CP060086.1).

\section{Phylogenetic analyses}

To explore the genetic diversity and phylogenetic relationships between JNQH-PA57 and other clinical $P$. aeruginosa strains, SNPs were called from 158 genomes of $P$. aeruginosa strains (including 157 publicly available complete genomes of clinically isolated P.aeruginosa strains from the PGDB and JNQH-PA57 from this study) using snippy v. 4.4.1 (https:/github.com/tseemann/snippy). Alignments were filtered for recombinations using Gubbins v. 2.4.1 [62] and core SNPs extracted using SNP-sites v. 2.5.1 [63]. An approximately-maximum-likelihood phylogenetic tree from alignments of nucleotide sequences was inferred with FastTree [64] and the visualization and annotation of the phylogenetic tree were carried out using an online tool Interactive Tree of Life (iTOL) (https://itol.embl.de/) [65].

\section{Genomic analysis}

Complete genome comparison of JNQH-PA57 with three wildly studied $P$. aeruginosa reference strains was carried out by BLASTn search using BRIG v0.95 [66]. GIs and prophages were labeled in their respective locations. Multiple sequence alignment analysis of $P$. aeruginosa JNQH-PA57 and PAO1, PA14 was performed using progressive Mauve with default setting [67]. GIs in the P. aeruginosa JNQHPA57 genome were predicted with the Island Viewer 4 server [25] based on the prediction method IslandPathDIMOB [68]. The online software PHASTER was used to predict the prophages in the genome of this strain [26].

COG comparison analysis was performed with VennDiagram in R-platform [69]. Proteins present exclusively in an individual strain and those shared between two or three strains were counted based on Mauve and COG blast analysis, and ultimately represented in Venn diagrams as reported previously [19].

Identification of putative ICE $\mathrm{INP}_{\mathrm{JN} 5}$ in the JNQH-PA57 genome was performed using a web-based tool ICEfinder (https://db-mml.sjtu.edu.cn/ICEfinder/ICEfinder. 
html) based on an updated database ICEberg 2.0 [28]. Visualizing comparative analysis of ICEs that belongs to PAPI-1 families and gene ortholog prediction was carried out with Easyfig v2.2.3 [70].

A set of genes related to the antibiotic resistance in $P$. aeruginosa $\mathrm{JNQH}-\mathrm{PA} 57$ were identified using the online CARD (https://card.mcmaster.ca/home) [71]. Identity and coverage thresholds were set to $90 \%$. The sequences of the resistance genes and OprD encoding gene were compared with reference strain PAO1 and those deposited at GenBank using BLASTn (http://www.ncbi.nih. gov/BLAST).

\section{Growth curve measurement, biofilm detection and colony morphology assay}

The growth curves of $P$. aeruginosa strains were measured via a microplate method. Overnight cultures of the strains were adjusted with fresh $\mathrm{LB}$ to $\mathrm{OD}_{600}$ of 0.01 and then transferred $(200 \mu \mathrm{l})$ into each well of a 96-well cell culture plate (Corstar, USA) with a cover. Then the microplate was incubated at $37^{\circ} \mathrm{C}$ for $24 \mathrm{~h}$ with being shaken automatically at one-minute intervals and optical density at $600 \mathrm{~nm}$ was determined every 20 min with a microplate reader (Thermo, USA).

Biofilm formation was assayed in 96-well plates with crystal violet staining as described previously [72]. Briefly, overnight cultures were adjusted with fresh LB to the same $\mathrm{OD}_{600}$ and then diluted in a fresh LB (1: 100). Further, $100 \mu \mathrm{l}$ of these diluted cultures were transferred into a new 96-well plate and incubated at $37{ }^{\circ} \mathrm{C}$ statically for $24 \mathrm{~h}$ with lid. Then the liquid cultures were discarded by suction, and the 96-well plate was washed with distilled $\mathrm{H}_{2} \mathrm{O}$ to remove media and unattached cell material. $125 \mu \mathrm{l} 0.1 \%$ crystal violet staining solution was added per well and incubated at room temperature for $15 \mathrm{~min}$, followed by removing the staining solution and washing the plate with $\mathrm{H}_{2} \mathrm{O}$. After the plates were dried at room temperature, $125 \mu \mathrm{l} 30 \%$ acetic acid solution was added to each well to destain the pellicle biofilm ring for $15 \mathrm{~min}$ at room temperature. The violet acetic acid solution was transferred into a fresh 96-well plate, and the absorbance was measured at $550 \mathrm{~nm}$.

Colony morphology of $P$. aeruginosa was detected by culturing the strains at $25^{\circ} \mathrm{C}$ on $1 \%$ tryptone agar plates supplemented with $20 \mu \mathrm{g} / \mathrm{mL}$ coomassie blue and $40 \mu \mathrm{g} /$ $\mathrm{mL}$ congo red [73] for 3 days.

\section{Gene-expression analysis}

For the gene-expression assays, the strains were grown overnight, then diluted in a fresh culture (1:100) and grown to the early exponential phase $\left(\mathrm{OD}_{600}=1.0\right)$ in LB at $37{ }^{\circ} \mathrm{C}$ in a shaking incubator. Total RNA was extracted using TRIpure reagent (Biofit, China) as described in the manual. Total RNA (500 ng) from all isolates was reverse transcribed into single-stranded cDNA using RT6 cDNA Synthesis Kit Ver 2 (TSINGKE, China). QRT-PCR experiments were carried out using $2 \times \mathrm{T} 5$ Fast qPCR Mix (SYBR Green I) (TSINGKE, China). Primers specific for the amplification of genes related to alginate biosynthesis ( $\operatorname{alg} U$ and $\operatorname{alg} D$ ), efflux proteins (mexB and mexY) were listed in additional file 9 (Table S9). And the amplifications were performed with the LineGene 9600 Plus system (BIOER, China). A ribosomal protein encoding gene, rpsL, was used as a reference gene for normalizing the expression levels of target genes [74]. Relative transcript levels were determined by the comparative standard curve method.

\section{Abbreviations \\ MDR: Multidrug-resistant; ICE: Integrative and conjugative element; GI: Genomic island; IS: Insertion sequence; NCBI: National Center for Biotechnology Information; MIC: Minimal inhibitory concentration; PBA: Phenylboronic acid; PGAP: Prokaryotic Genome Annotation Pipeline; CDS: Coding sequences; SNP: Single nucleotide polymorphism; BRIG: BLAST Ring Image Generator; CARD: Comprehensive Antibiotic Resistance Database; ORFs: Open reading frames; T4SS: Type IV secretion system; COG: Clusters of Orthologous Groups; qRT-PCR: Quantitative reverse transcription-PCR; AMR: Antimicrobial resistance; PDC: Pseudomonas-derived cephalosporinase; RND: Resistance-nodulation-division; MHA: Mueller-Hinton agar; CLSI: Clinical and Laboratory Standards Institute; iTOL: Interactive Tree of Life}

\section{Supplementary Information}

The online version contains supplementary material available at https://doi. org/10.1186/s12866-021-02203-4.

Additional file 1: Table S1. List of the genomic features of mucoid strain $P$. aeruginosa JNQH-PA57 revealed from the complete genome (this study) and those representative $P$. aeruginosa clinical isolates listed in the Pseudomonas Genome Database (PGDB).

Additional file 2: Table S2. Strains of clinically isolated $P$. aeruginosa used in this study downloaded from Pseudomonas genome database based on the availability of complete genomes

Additional file 3: Table S3. Detail information of four large insertion regions which absent in $P$. aeruginosa $\mathrm{PAO} 1$ genome but exist in $P$. aeruginosa $\mathrm{JNQH}-\mathrm{PQ} 57$ genome

Additional file 4: Table S4. Detail information of Gls identified in P. aeruginosa JNQH-PQ57 genome with IslandPath-DIMOD method

Additional file 5: Table S5. Detail information of prophages identified in $P$. aeruginosa JNQH-PQ57 genome

Additional file 6: Table S6. Detail information of ICE JNPA57

Additional file 7: Table S7. COGs uniquely identified in $P$. aeruginosa JNQH-PA057 but absent in PAO1, ATCC 27853 and PA14

Additional file 8: Table S8. AMR profile of the $P$. aeruginosa $\mathrm{JNQH}$ PA57

Additional file 9: Table S9. Primers used for qRT-PCR ( $f$, forward, sense, and $r$, reverse, antisense)

\section{Acknowledgments}

We would like to sincerely and genuinely thank Prof. Dr. Chuiqing Ma and Prof.Dr. Chao Gao (State Key Laboratory of Microbial Technology, Shandong University, Qingdao 266237, People's Republic of China) for kindly providing the reference strain $P$. aeruginosa $\mathrm{PAO} 1$ and $P$. aeruginosa PA14.

\section{Authors' contributions}

YW was the principal investigator, conceived and designed the project, analyzed and interpreted the data, and contributed to writing the manuscript. MH provided bioinformatics analysis for the project, designed 
the experiments in collaboration with WY, and wrote parts of the manuscript. WM supervised the study and critically revised the manuscript. $\mathrm{XD}, \mathrm{XL}$ and $C F$ provided resources and performed experiments described in this study. All authors read and approved the final manuscript.

\section{Funding}

This research was supported by the Jinan Clinical Medicine Technology Innovation Program, grant number 202019129

\section{Availability of data and materials}

The dataset of complete genome of $P$. aeruginosa JNQH-PA57 sequence has been deposited to National Center Biotechnology Information with the accession number CP060086 (https://www.ncbi.nlm.nih.gov/nuccore/NZ_CP060086.1).

\section{Declarations}

Ethics approval and consent to participate Not applicable.

\section{Consent for publication}

Not applicable.

\section{Competing interests}

The authors declare that they have no competing interests.

Received: 5 March 2021 Accepted: 19 April 2021

Published online: 01 May 2021

\section{References}

1. Lund-Palau H, Turnbull AR, Bush A, Bardin E, Cameron L, Soren O, et al. Pseudomonas aeruginosa infection in cystic fibrosis: pathophysiological mechanisms and therapeutic approaches. Expert Rev Respir Med. 2016;10(6): 685-97. https://doi.org/10.1080/17476348.2016.1177460.

2. Ahator SD, Zhang L. Small is mighty-chemical communication systems in Pseudomonas aeruginosa. Annu Rev Microbiol. 2019;73(1):559-78. https://doi. org/10.1146/annurev-micro-020518-120044.

3. Kang $\mathrm{Cl}$, Kim SH, Kim HB, Park SW, Choe YJ, Oh MD, et al. Pseudomonas aeruginosa bacteremia: risk factors for mortality and influence of delayed receipt of effective antimicrobial therapy on clinical outcome. Clin Infect Dis. 2003;37(6):745-51. https://doi.org/10.1086/377200.

4. Miyoshi-Akiyama T, Tada T, Ohmagari N, Hung NV, Tharavichitkul P, Pokhrel $\mathrm{BM}$, et al. Emergence and spread of epidemic multidrug-resistant Pseudomonas aeruginosa. Genome Biol Evol. 2017;9(12):3238-45. https://doi. org/10.1093/gbe/evx243.

5. Telling K, Laht M, Brauer A, Remm M, Kisand V, Maimets M, et al. Multidrug resistant Pseudomonas aeruginosa in Estonian hospitals. BMC Infect Dis. 2018;18(1):513. https://doi.org/10.1186/s12879-018-3421-1.

6. $\quad$ Ding C, Yang Z, Wang J, Liu X, Cao Y, Pan Y, et al. Prevalence of Pseudomonas aeruginosa and antimicrobial-resistant Pseudomonas aeruginosa in patients with pneumonia in mainland China: a systematic review and meta-analysis. Int J Infect Dis. 2016;49:119-28. https://doi.org/1 0.1016/j.jijid.2016.06.014.

7. Ding Y, Teo JWP, Drautz-Moses DI, Schuster SC, Givskov M, Yang L. Acquisition of resistance to carbapenem and macrolide-mediated quorum sensing inhibition by Pseudomonas aeruginosa via ICE $\mathrm{Tn}_{4371}$ 6385. Commun Biol. 2018;1(1):57. https://doi.org/10.1038/s42003-018-0064-0.

8. Petrova A, Feodorova Y, Miteva-Katrandzhieva T, Petrov M, Murdjeva M. First detected OXA-50 carbapenem-resistant clinical isolates Pseudomonas aeruginosa from Bulgaria and interplay between the expression of main efflux pumps, OprD and intrinsic AmpC. J Med Microbiol. 2019;68(12):172331. https://doi.org/10.1099/jmm.0.001106.

9. Malhotra S, Hayes D Jr, Wozniak DJ. Mucoid Pseudomonas aeruginosa and regional inflammation in the cystic fibrosis lung. J Cyst Fibros. 2019;18(6): 796-803. https://doi.org/10.1016/j.jcf.2019.04.009.

10. Subedi D, Vijay AK, Kohli GS, Rice SA, Willcox M. Comparative genomics of clinical strains of Pseudomonas aeruginosa strains isolated from different geographic sites. Sci Rep. 2018;8(1):15668. https://doi.org/10.1038/s41598-01 8-34020-7.

11. Klockgether J, Cramer N, Wiehlmann L, Davenport CF, Tummler B. Pseudomonas aeruginosa genomic structure and diversity. Front Microbiol. 2011;2:150. https://doi.org/10.3389/fmicb.2011.00150.
12. Kung VL, Ozer EA, Hauser AR. The accessory genome of Pseudomonas aeruginosa. Microbiol Mol Biol Rev. 2010;74(4):621-41. https://doi.org/10.112 8/MMBR.00027-10

13. Kawalek A, Kotecka K, Modrzejewska M, Gawor J, Jagura-Burdzy G, Bartosik AA. Genome sequence of Pseudomonas aeruginosa PAO1161, a PAO1 derivative with the ICEPae1161 integrative and conjugative element. BMC Genomics. 2020;21(1):14. https://doi.org/10.1186/s12864-019-6378-6.

14. Wang D, Hildebrand F, Ye L, Wei Q, Ma LZ. Genome sequence of mucoid Pseudomonas aeruginosa strain FRD1. Genome Announc. 2015;3(2). https:// doi.org/10.1128/genomeA.00376-15.

15. Norman A, Ciofu O, Amador Cl, Hoiby N, Jelsbak L. Genome sequence of Pseudomonas aeruginosa strain DK1-NH57388A, a stable mucoid cystic fibrosis isolate. Genome Announc. 2016;4(1). https://doi.org/10.1128/ genomeA.00008-16.

16. Winsor GL, Griffiths EJ, Lo R, Dhillon BK, Shay JA, Brinkman FS. Enhanced annotations and features for comparing thousands of Pseudomonas genomes in the Pseudomonas genome database. Nucleic Acids Res. 2016; 44(D1):D646-53. https://doi.org/10.1093/nar/gkv1227.

17. Roy PH, Tetu SG, Larouche A, Elbourne L, Tremblay S, Ren $\mathrm{Q}$, et al. Complete genome sequence of the multiresistant taxonomic outlier Pseudomonas aeruginosa PA7. PLoS One. 2010;5(1):e8842. https://doi.org/1 0.1371/journal.pone.0008842.

18. Stover CK, Pham XQ, Erwin AL, Mizoguchi SD, Warrener P, Hickey MJ, et al. Complete genome sequence of Pseudomonas aeruginosa PAO1, an opportunistic pathogen. Nature. 2000;406(6799):959-64. https://doi.org/10.1 038/35023079

19. Cao H, Lai Y, Bougouffa S, Xu Z, Yan A. Comparative genome and transcriptome analysis reveals distinctive surface characteristics and unique physiological potentials of Pseudomonas aeruginosa ATCC 27853. BMC Genomics. 2017:18(1):459. https://doi.org/10.1186/s12864-017-3842-z.

20. Jeukens J, Boyle B, Kukavica-Ibrulj I, Ouellet MM, Aaron SD, Charette SJ, et al. Comparative genomics of isolates of a Pseudomonas aeruginosa epidemic strain associated with chronic lung infections of cystic fibrosis patients. PLoS One. 2014:9(2):e87611. https://doi.org/10.1371/journal.pone.0087611.

21. Damkiaer S, Yang L, Molin S, Jelsbak L. Evolutionary remodeling of global regulatory networks during long-term bacterial adaptation to human hosts. Proc Natl Acad Sci U S A. 2013;110(19):7766-71. https://doi.org/10.1073/pna S. 1221466110

22. Cain AK, Nolan LM, Sullivan GJ, Whitchurch CB, Filloux A, Parkhill J. Complete Genome Sequence of Pseudomonas aeruginosa Reference Strain PAK. Microbiol Resour Announc. 2019;8(41). https://doi.org/10.1128/MRA. 00865-19.

23. He J, Baldini RL, Ziel ED, saucier $M$, Zhang $Q$, Liberati NT, et al. The broad host range pathogen Pseudomonas aeruginosa strain PA14 carries two pathogenicity islands harboring plant and animal virulence genes. Proc Natl Acad Sci U S A. 2004;101(8):2530-5. https://doi.org/10.1073/ pnas.0304622101.

24. Lee DG, Urbach JM, Wu G, Liberati NT, Feinbaum RL, Miyata S, et al. Genomic analysis reveals that Pseudomonas aeruginosa virulence is combinatorial. Genome Biol. 2006;7(10):R90. https://doi.org/10.1186/gb-2 006-7-10-r90.

25. Bertelli C, Laird MR, Williams KP. Simon Fraser University research computing G, Lau BY, Hoad G, et al. IslandViewer 4: expanded prediction of genomic islands for larger-scale datasets. Nucleic Acids Res. 2017;45(W1): W30-W5. https://doi.org/10.1093/nar/gkx343.

26. Arndt D, Grant JR, Marcu A, Sajed T, Pon A, Liang Y, et al. PHASTER: a better, faster version of the PHAST phage search tool. Nucleic Acids Res. 2016; 44(W1):W16-21. https://doi.org/10.1093/nar/gkw387.

27. Hill DF, Short NJ, Perham RN, Petersen GB. DNA sequence of the filamentous bacteriophage Pf1. J Mol Biol. 1991;218(2):349-64. https://doi. org/10.1016/0022-2836(91)90717-k

28. Liu M, Li X, Xie Y, Bi D, Sun J, Li J, et al. ICEberg 2.0: an updated database of bacterial integrative and conjugative elements. Nucleic Acids Res. 2019; 47(D1):D660-D5. https://doi.org/10.1093/nar/gky1123.

29. Klockgether J, Reva O, Larbig K, Tummler B. Sequence analysis of the mobile genome island pKLC102 of Pseudomonas aeruginosa C. J Bacteriol. 2004;186(2):518-34. https://doi.org/10.1128/jb.186.2.518-534.2 004.

30. Qiu X, Gurkar AU, Lory S. Interstrain transfer of the large pathogenicity island (PAPI-1) of Pseudomonas aeruginosa. Proc Natl Acad Sci U S A. 2006; 103(52):19830-5. https://doi.org/10.1073/pnas.0606810104. 
31. Mathee K, Narasimhan G, Valdes C, Qiu X, Matewish JM, Koehrsen M, et al. Dynamics of Pseudomonas aeruginosa genome evolution. Proc Natl Acad Sci U S A. 2008;105(8):3100-5. https://doi.org/10.1073/pnas.0711982105.

32. Mavrodi DV, Loper JE, Paulsen IT, Thomashow LS. Mobile genetic elements in the genome of the beneficial rhizobacterium Pseudomonas fluorescens Pf5. BMC Microbiol. 2009;9(1):8. https://doi.org/10.1186/1471-2180-9-8.

33. Johnson CM, Grossman AD. Integrative and conjugative elements (ICEs): what they do and how they work. Annu Rev Genet. 2015;49(1):577-601. https://doi.org/10.1146/annurev-genet-112414-055018.

34. Carter MQ, Chen J, Lory S. The Pseudomonas aeruginosa pathogenicity island PAPI-1 is transferred via a novel type IV pilus. J Bacteriol. 2010;192(13): 3249-58. https://doi.org/10.1128/JB.00041-10.

35. Hirano N, Muroi T, Takahashi H, Haruki M. Site-specific recombinases as tools for heterologous gene integration. Appl Microbiol Biotechnol. 2011;92(2): 227-39. https://doi.org/10.1007/s00253-011-3519-5.

36. Hwang LC, Vecchiarelli AG, Han YW, Mizuuchi M, Harada Y, Funnell BE, et al. ParA-mediated plasmid partition driven by protein pattern self-organization. EMBO J. 2013;32(9):1238-49. https://doi.org/10.1038/emboj.2013.34.

37. Martin DW, Schurr MJ, Mudd MH, Govan JR, Holloway BW, Deretic V. Mechanism of conversion to mucoidy in Pseudomonas aeruginosa infecting cystic fibrosis patients. Proc Natl Acad Sci U S A. 1993;90(18):8377-81. https://doi.org/10.1073/pnas.90.18.8377.

38. Yin $Y$, Damron FH, Withers TR, Pritchett $\mathrm{CL}$, Wang $X$, Schurr MJ, et al. Expression of mucoid induction factor MucE is dependent upon the alternate sigma factor AlgU in Pseudomonas aeruginosa. BMC Microbiol. 2013;13(1):232. https://doi.org/10.1186/1471-2180-13-232.

39. Hay ID, Gatland K, Campisano A, Jordens JZ, Rehm BH. Impact of alginate overproduction on attachment and biofilm architecture of a supermucoid Pseudomonas aeruginosa strain. Appl Environ Microbiol. 2009;75(18):6022-5. https://doi.org/10.1128/AEM.01078-09.

40. Mathee K, Ciofu O, Sternberg C, Lindum PW, Campbell JIA, Jensen P, et al. Mucoid conversion of Pseudomonas aeruginosa by hydrogen peroxide: a mechanism for virulence activation in the cystic fibrosis lung. Microbiology (Reading). 1999;145(Pt 6):1349-57. https://doi.org/10.1099/13500872-1456-1349.

41. Ryall B, Carrara M, Zlosnik JE, Behrends V, Lee X, Wong Z, et al. The mucoid switch in Pseudomonas aeruginosa represses quorum sensing systems and leads to complex changes to stationary phase virulence factor regulation. PLoS One. 2014;9(5):e96166. https://doi.org/10.1371/journal.pone.0096166.

42. Hoiby N, Bjarnsholt T, Givskov M, Molin S, Ciofu O. Antibiotic resistance of bacterial biofilms. Int J Antimicrob Agents. 2010;35(4):322-32. https://doi. org/10.1016/j.jijantimicag.2009.12.011.

43. Hoiby N, Ciofu O, Bjarnsholt T. Pseudomonas aeruginosa biofilms in cystic fibrosis. Future Microbiol. 2010;5(11):1663-74. https://doi.org/1 $0.2217 / \mathrm{fmb} .10 .125$

44. Jacoby GA. AmpC beta-lactamases. Clin Microbiol Rev. 2009;22(1):161-82, Table of Contents. https://doi.org/10.1128/CMR.00036-08.

45. Girlich D, Naas T, Nordmann P. Biochemical characterization of the naturally occurring oxacillinase OXA-50 of Pseudomonas aeruginosa. Antimicrob Agents Chemother. 2004;48(6):2043-8. https://doi.org/10.1128/AAC.48.6.2 043-2048.2004.

46. Fang ZL, Zhang LY, Huang YM, Qing Y, Cao KY, Tian GB, et al. OprD mutations and inactivation in imipenem-resistant Pseudomonas aeruginosa isolates from China. Infect Genet Evol. 2014;21:124-8. https://doi.org/10.101 6/j.meegid.2013.10.027

47. Kao CY, Chen SS, Hung KH, Wu HM, Hsueh PR, Yan JJ, et al. Overproduction of active efflux pump and variations of OprD dominate in imipenem-resistant Pseudomonas aeruginosa isolated from patients with bloodstream infections in Taiwan. BMC Microbiol. 2016;16(1):107. https://doi.org/10.1186/s12866-016-0719-2.

48. Shu JC, Kuo AJ, Su LH, Liu TP, Lee MH, Su IN, et al. Development of carbapenem resistance in Pseudomonas aeruginosa is associated with OprD polymorphisms, particularly the amino acid substitution at codon 170. J Antimicrob Chemother. 2017;72(9):2489-95. https://doi.org/10.1093/jac/dkx158.

49. Ochs MM, Bains M, Hancock RE. Role of putative loops 2 and 3 in imipenem passage through the specific porin OprD of Pseudomonas aeruginosa. Antimicrob Agents Chemother. 2000;44(7):1983-5. https://doi.org/10.112 8/aac.44.7.1983-1985.2000.

50. Lister PD, Wolter DJ, Hanson ND. Antibacterial-resistant Pseudomonas aeruginosa: clinical impact and complex regulation of chromosomally encoded resistance mechanisms. Clin Microbiol Rev. 2009;22(4):582-610. https://doi.org/10.1128/CMR.00040-09.
51. Riera E, Cabot G, Mulet X, Garcia-Castillo M, del Campo R, Juan C, et al. Pseudomonas aeruginosa carbapenem resistance mechanisms in Spain: impact on the activity of imipenem, meropenem and doripenem. J Antimicrob Chemother. 2011;66(9):2022-7. https://doi. org/10.1093/jac/dkr232.

52. Nikaido H, Pages JM. Broad-specificity efflux pumps and their role in multidrug resistance of gram-negative bacteria. FEMS Microbiol Rev. 2012; 36(2):340-63. https://doi.org/10.1111/j.1574-6976.2011.00290.x.

53. Terzi HA, Kulah C, Ciftci $\mathrm{H}$. The effects of active efflux pumps on antibiotic resistance in Pseudomonas aeruginosa. World J Microbiol Biotechnol. 2014; 30(10):2681-7. https://doi.org/10.1007/s11274-014-1692-2.

54. Poole K, Srikumar R. Multidrug efflux in Pseudomonas aeruginosa: components, mechanisms and clinical significance. Curr Top Med Chem. 2001;1(1):59-71. https://doi.org/10.2174/1568026013395605.

55. Clinical and Laboratory Standards Institute. Performance standards for antimicrobial susceptibility testing, thirdty informational supplement, M100S30. Wayne: Clinical and Laboratory Standards Institute; 2020.

56. Tsakris A, Poulou A, Pournaras S, Voulgari E, Vrioni G, Themeli-Digalaki K, et al. A simple phenotypic method for the differentiation of metallo-betalactamases and class a KPC carbapenemases in Enterobacteriaceae clinical isolates. J Antimicrob Chemother. 2010;65(8):1664-71. https://doi.org/10.1 093/jac/dkq210

57. Wick RR, Judd LM, Gorrie CL, Holt KE. Unicycler: resolving bacterial genome assemblies from short and long sequencing reads. PLoS Comput Biol. 2017; 13(6):e1005595. https://doi.org/10.1371/journal.pcbi.1005595.

58. Walker BJ, Abeel T, Shea T, Priest M, Abouelliel A, Sakthikumar S, et al. Pilon: an integrated tool for comprehensive microbial variant detection and genome assembly improvement. PLoS One. 2014;9(11):e112963. https://doi. org/10.1371/journal.pone.0112963.

59. Tatusova T, DiCuccio M, Badretdin A, Chetvernin V, Nawrocki EP, Zaslavsky L, et al. NCBI prokaryotic genome annotation pipeline. Nucleic Acids Res. 2016 44(14):6614-24. https://doi.org/10.1093/nar/gkw569.

60. Lowe TM, Chan PP. tRNAscan-SE on-line: integrating search and context for analysis of transfer RNA genes. Nucleic Acids Res. 2016;44(W1):W54-7. https://doi.org/10.1093/nar/gkw413

61. Lagesen $\mathrm{K}$, Hallin P, Rodland EA, Staerfeldt HH, Rognes T, Ussery DW. RNAmmer: consistent and rapid annotation of ribosomal RNA genes. Nucleic Acids Res. 2007;35(9):3100-8. https://doi.org/10.1093/nar/gkm160.

62. Croucher NJ, Page AJ, Connor TR, Delaney AJ, Keane JA, Bentley SD, et al. Rapid phylogenetic analysis of large samples of recombinant bacterial whole genome sequences using Gubbins. Nucleic Acids Res. 2015;43(3):e15. https://doi.org/10.1093/nar/gku1196.

63. Page AJ, Taylor B, Delaney AJ, Soares J, Seemann T, Keane JA, et al. SNPsites: rapid efficient extraction of SNPs from multi-FASTA alignments. Microb Genom. 2016;2(4):e000056. https://doi.org/10.1099/mgen.0.000056.

64. Price MN, Dehal PS, Arkin AP. FastTree: computing large minimum evolution trees with profiles instead of a distance matrix. Mol Biol Evol. 2009;26(7): 1641-50. https://doi.org/10.1093/molbev/msp077.

65. Letunic I, Bork P. Interactive tree of life (iTOL) v4: recent updates and new developments. Nucleic Acids Res. 2019;47(W1):W256-W9. https://doi.org/1 $0.1093 /$ nar/gkz239.

66. Alikhan NF, Petty NK, Ben Zakour NL, Beatson SA. BLAST ring image generator (BRIG): simple prokaryote genome comparisons. BMC Genomics. 2011;12(1):402. https://doi.org/10.1186/1471-2164-12-402.

67. Darling AE, Mau B, Perna NT. progressiveMauve: multiple genome alignment with gene gain, loss and rearrangement. PLoS One. 2010;5(6): e11147. https://doi.org/10.1371/journal.pone.0011147.

68. Hsiao W, Wan I, Jones SJ, Brinkman FS. IslandPath: aiding detection of genomic islands in prokaryotes. Bioinformatics. 2003;19(3):418-20. https:// doi.org/10.1093/bioinformatics/btg004.

69. Chen H, Boutros PC. VennDiagram: a package for the generation of highlycustomizable Venn and Euler diagrams in R. BMC Bioinformatics. 2011;12(1): 35. https://doi.org/10.1186/1471-2105-12-35.

70. Sullivan MJ, Petty NK, Beatson SA. Easyfig: a genome comparison visualizer. Bioinformatics. 2011;27(7):1009-10. https:/doi.org/10.1093/bioinformatics/btr039.

71. Jia B, Raphenya AR, Alcock B, Waglechner N, Guo P, Tsang KK, et al. CARD 2017: expansion and model-centric curation of the comprehensive antibiotic resistance database. Nucleic Acids Res. 2017:45(D1):D566-D73. https://doi.org/10.1093/nar/gkw1004.

72. O'Toole GA. Microtiter dish biofilm formation assay. J Vis Exp. 2011:47(47). https://doi.org/10.3791/2437. 
73. Dietrich LE, Okegbe C, Price-Whelan A, Sakhtah H, Hunter RC, Newman DK. Bacterial community morphogenesis is intimately linked to the intracellular redox state. J Bacteriol. 2013;195(7):1371-80. https://doi. org/10.1128/JB.02273-12.

74. EL Amin N, Giske CG, Jalal S, Keijser B, Kronvall G, Wretlind B. Carbapenem resistance mechanisms in Pseudomonas aeruginosa: alterations of porin OprD and efflux proteins do not fully explain resistance patterns observed in clinical isolates. APMIS. 2005;113(3):187-96. https://doi.org/10.1111/j.16000463.2005.apm1130306.X.

\section{Publisher's Note}

Springer Nature remains neutral with regard to jurisdictional claims in published maps and institutional affiliations.

Ready to submit your research? Choose BMC and benefit from:

- fast, convenient online submission

- thorough peer review by experienced researchers in your field

- rapid publication on acceptance

- support for research data, including large and complex data types

- gold Open Access which fosters wider collaboration and increased citations

- maximum visibility for your research: over $100 \mathrm{M}$ website views per year

At $\mathrm{BMC}$, research is always in progress.

Learn more biomedcentral.com/submissions 